\title{
Three-Dimensional Computed Tomography Follow-Up on Spontaneous Bone Healing after Excision of Periorbital Dermoid Cyst: A Case Report
}

\author{
Hannara Park (D), Jaemin Seong (D), Hyochun Park (D), Hyeonjung Yeo (D) \\ Department of Plastic and Reconstructive Surgery, Daegu Fatima Hospital, Daegu, Korea
}

\begin{abstract}
Dermoid cysts are among the most common periorbital and orbital tumors presenting in childhood. Several studies have shown that dermoid cysts may deform adjacent bones; however, few studies have followed the course of bone regeneration after the excision of a cyst. We report a case of a 29-month-old female infant who presented with a palpable mass over her right lateral eyebrow. Ultrasonography findings suggested a dermoid cyst, and computed tomography revealed a $2.1-\mathrm{cm}$ round bony defect in the right zygoma. After surgical excision of the dermoid cyst, we successfully applied fibrin sealant to eliminate the dead space. Pathological findings showed nodular lesions with flat cystic walls containing lamellated keratin and sebaceous glands, supporting the diagnosis of dermoid cyst. At 6 months postoperatively, a follow-up computed tomography scan confirmed complete recovery of the bone defect in the right zygoma.
\end{abstract}

Keywords: Dermoid cyst; Fibrin tissue adhesive; Bone regeneration

\section{Introduction}

Dermoid cysts arise from ectodermal remnants, sequestered due to the incomplete fusion of the neuroectoderm during the formation of the neural tube, and can be differentiated from both the ectoderm and mesoderm [1]. Histologically, dermoid cysts have a fibrous wall lined by keratinizing squamous epithelium, and contain skin adnexa such as hair follicles, smooth muscles, and sweat and sebaceous glands [2]. Dermoid cysts are typically slow-growing and benign tumors containing developmentally mature tissues $[2,3]$. Though dermoid cysts may occur anywhere in the body, they are most commonly found in the periorbital lateral eyebrow area in children [3].

Dermoid cysts may lead to inflammatory responses as well as abnormal changes in the underlying bone, such as pressure erosions, tunnels, craters, and clefts [4]. These changes may then create a dead space, leading to an undesirable aesthetic appearance, or they may lead to adverse outcomes after surgical intervention, for example seroma formation and delayed wound healing. Therefore, in addition to completely removing the dermoid cyst, deformities in the adjacent bone need to be reconstructed.

Many studies have shown that dermoid cysts might abnormally deform the adjacent bone; however, only few studies have followed the course of bone regeneration after excision of the cyst. Here, we report a case of successful excision of a dermoid cyst on the right lateral eyebrow in a 29 -month-old female infant, and complete regeneration of the bone defect caused by the dermoid cyst on the right zygoma, follow-
Case Report

Received: March 28, 2021

Revised: June 15, 2021

Accepted: June 20, 2021

\section{Corresponding author:} Hannara Park, M.D

Department of Plastic and Reconstructive Surgery, Daegu Fatima Hospital, 99 Ayang-ro, Dong-gu, Daegu 41199, Korea

Tel: +82-53-940-7340

Fax: +82-53-940-7344

E-mail: hannara_@hanmail.net

This is an Open Access article distributed under the terms of the Creative Commons Attribution Non-Commercial License (https://creativecommons.org/licenses/by-nc/4.0/) which permits unrestricted non-commercial use, distribution, and reproduction in any medium, provided the original work is properly cited.

C) 2021 Korean Wound Management Society 
ing the application of a fibrin sealant (Tisseel; Baxter Healthcare Corp., Westlake Village, CA, USA). The study was approved by the Institutional Review Board of Daegu Fatima Hospital (IRB No. DFE20ORIO088) and performed in accordance with the principles of the Declaration of Helsinki. Written informed consent was obtained from the parents for the use of the images.

\section{Case}

A 29-month-old female infant presented with a palpable mass over her right lateral eyebrow. This had first been identified by her family when the child was 27 months old. According to the medical history obtained from her parents, she had not evidenced any neurological symptoms or visual compromise.

Ultrasonography revealed a complex cyst-like lesion, measuring approximately $2.1 \mathrm{~cm}$ in size, in contact with the right zygoma bone outside the orbital rim. The lack of an obvious increase in blood flow in the lesion strongly suggested a dermoid cyst (Fig. 1). Three-dimensional computed tomography (CT) showed a mass in the right temporal area and a round defect $2.1 \mathrm{~cm}$ in diameter in the right zygoma, surrounded by a well-defined margin (Fig. 2).

Complete surgical excision of the mass was performed under general anesthesia. The boundary of the tumor was marked using gentian violet, and an elliptical excision line was planned to position the postoperative scar on relaxed skin ten-

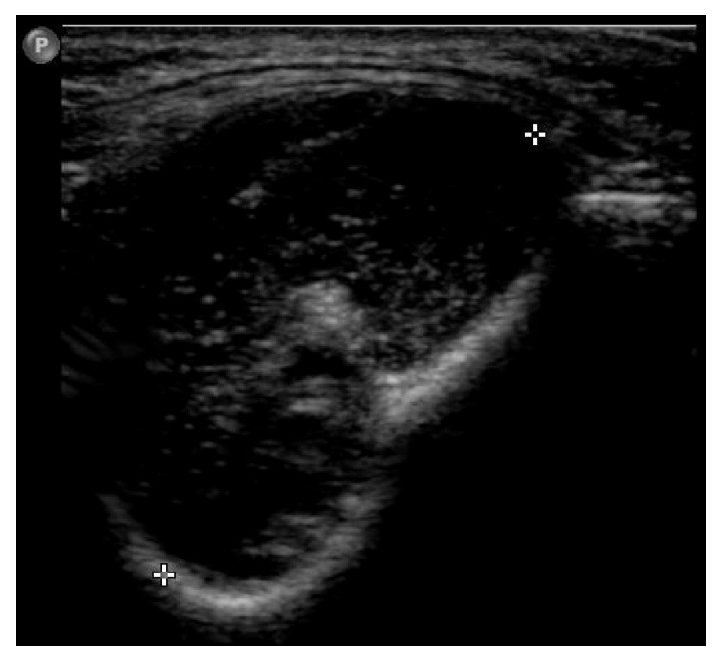

Fig. 1. Preoperative ultrasonography of the mass. Ultrasonography image showing a complex cyst-like lesion, measuring approximately $2.1 \mathrm{~cm}$ in size, in contact with the right zygoma bone outside the orbital rim and. No significant increase in blood flow in the lesion is observed. sion lines. An incision was made through the inferior margin of the lateral ends of the patient's eyebrows, and the tissue surrounding the mass was peeled back using Metzenbaum scissors to prevent tissue damage. The mass adherent to the temporoparietalis muscle and the frontal bone was completely excised to avoid cyst rupture (Fig. 3A). After excision of the mass, the surrounding bony defect was observed. A normal saline solution was used for intraoperative wound irrigation, and $2 \mathrm{~mL}$ of Tisseel was applied to eliminate the dead space and stimulate wound healing. The subcutaneous and epider-
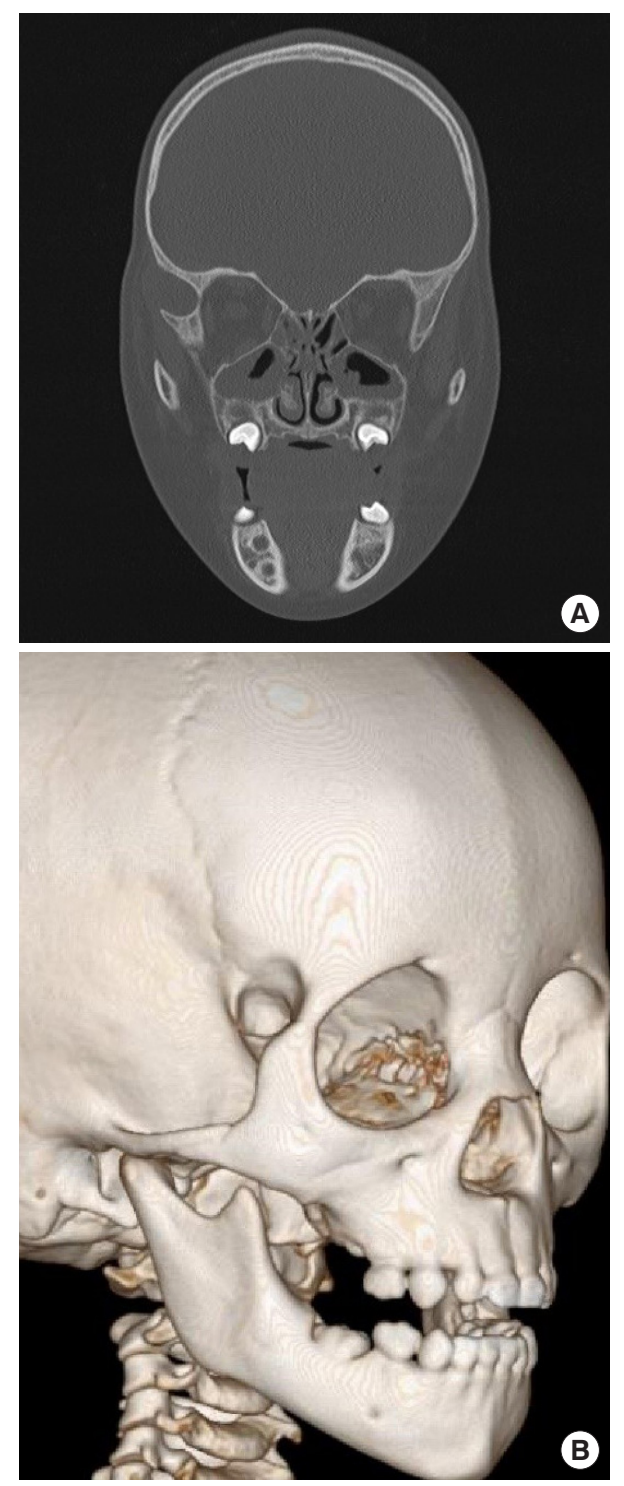

Fig. 2. Preoperative computed tomography image of facial bone. (A, B) Preoperative computed tomography scan demonstrating bone defect in the right zygoma. 
mal layers were then sutured using 5-0 polydioxanone and 6-0 nylon sutures, respectively (Fig. 3B).

The excised mass was pathologically diagnosed as a dermoid cyst (Fig. 4). There were no postoperative complications, and the incisions healed with a good aesthetic outcome. At 6
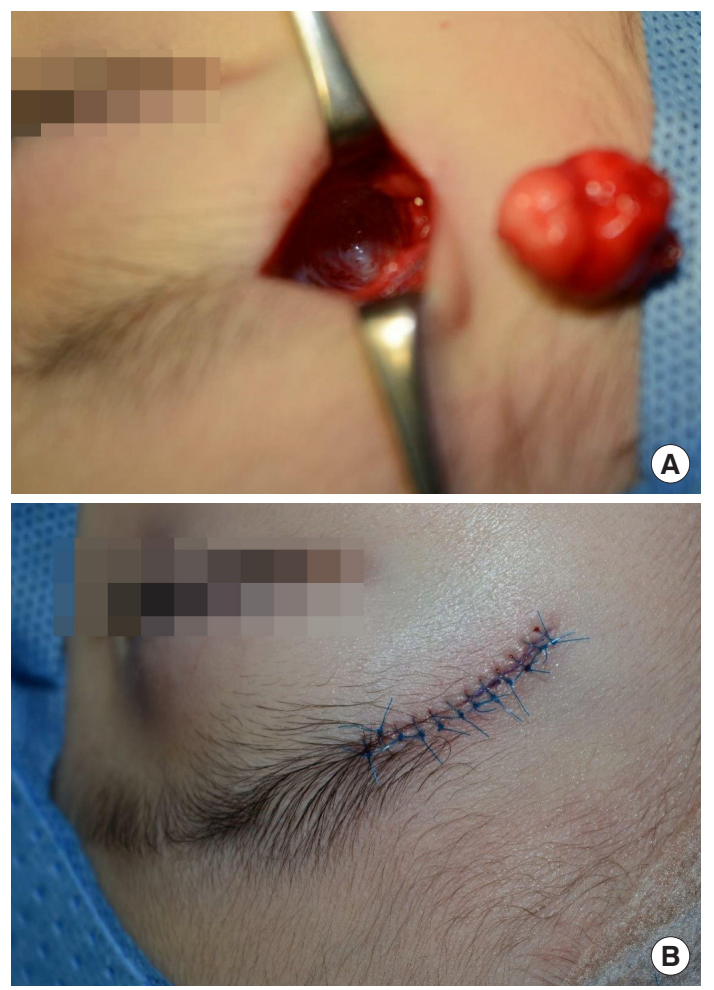

Fig. 3. Intraoperative photographic findings. (A) Intraoperative photograph showing bony defect surrounding the cyst after excision of the mass. (B) Immediate postoperative photography.

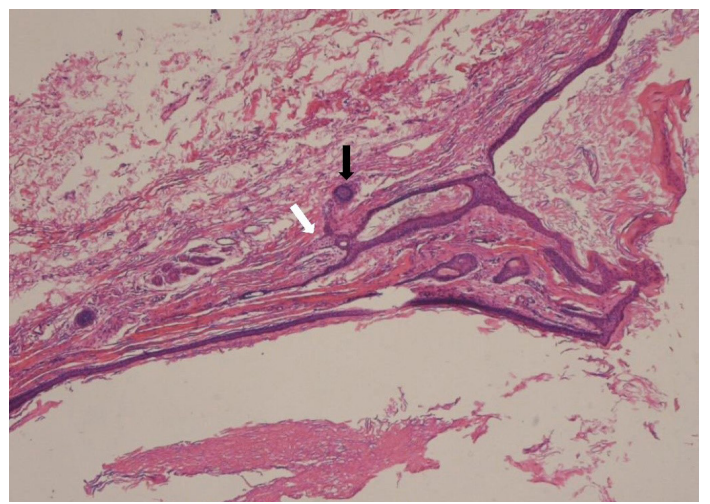

Fig. 4. Biopsy image of the mass. Histopathologic examination of the excised mass. Low magnification $(x 40)$ of H\&E stained tissue showing stratified, squamous epithelium-lined, mostly flat cystic wall with lamellated keratin contents. Skin adnexa including the sebaceous glands (white arrow) and the hair follicle (black arrow) are continuous with the epithelium of the cyst. months postoperatively, follow-up CT revealed the recovery of bone erosion (Fig. 5).

\section{Discussion}

Although dermoid cysts may occur anywhere in the body, their prevalence in the head and neck region is approximately $7.0 \%$, out of which ocular and orbital lesions account for more than $50 \%[4,5]$. Ten percent of orbital dermoid cysts occur within the orbit, mostly in the upper outer quadrant of the orbit [6].
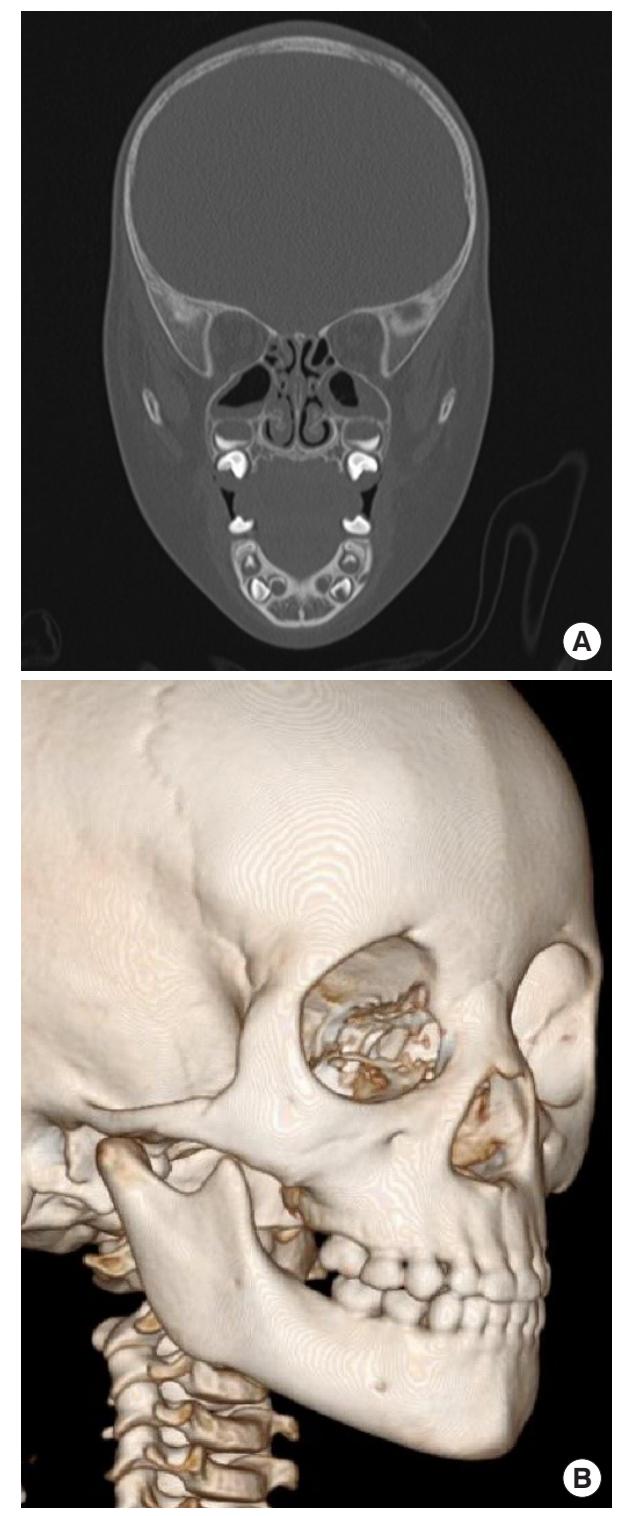

Fig. 5. Postoperative computed tomography image of facial bone. (A, B) Six-month postoperative computed tomography scan showing complete bone regeneration. 
Orbital dermoids result from the sequestration of the ectoderm within the suture lines of the orbital bones during formation of the neural tube. Dermoid cysts grow slowly, eroding the adjacent bones and effectively changing the surrounding tissue. According to a previous study, the bone of the lateral orbital wall and the superotemporal angle of the orbit develop abnormal bone texture and exhibit the following irregularities, often in combination: pressure erosion, a tunnel or canal through the lateral wall, a blind pit or crater, or a cleft [5]. The location, size, and depth of the accompanying bone abnormalities can be assessed by imaging techniques such as ultrasonography, CT, and magnetic resonance imaging. Imaging is also useful for evaluating postoperative outcomes [7].

Surgical excision is recommended for dermoid cysts, since ruptures may result in inflammation and granulation reactions due to the extrusion of the keratinous contents of the cyst, and can subsequently lead to symptoms such as proptosis, visual field defects, and strabismus in the periorbital region [8]. However, after removal of a dermoid cyst, the remaining dead space created by the accompanying bone abnormalities may lead to adverse surgical outcomes such as bleeding, seroma formation, and delayed wound healing, along with secondary problems such as inflammation, open wounds, and tissue necrosis.

To eliminate the dead space created by the excision of dermoid cysts, various methods using orbital fat flap, hydroxyapatite particulate artificial bone, and demineralized bone matrix have been suggested $[9,10]$. Tisseel, used in this study, is a twocomponent fibrin sealant that is vapor-heated and contains four substances: sealer protein concentrate (human), fibrinolysis inhibitor solution (bovine), thrombin (human), and calcium chloride. Fibrinogen, a constituent of the fibrin sealant, is transformed into fibrin in the presence of thrombin and calcium, promoting infection control and new tissue development through the release of platelet aggregation and growth factors. Aprotinin in the fibrin sealant, is an antiplasmin responsible for inhibiting premature fibrinolysis of the fibrin polymer.

Furthermore, fibrin sealants reduce the incidence of seroma formation by reducing the dead space created by the accompanying bone defects, inducing tissue adhesion, and reducing the blood or serous fluid secreted from the surface of the tissue by blocking the lymphatic and vascular tissue channels [1115]. Additionally, fibrin sealants promote bone healing by supporting the proliferation of mesenchymal and endothelial cells. This leads to increased formation of granulation tissue and deposition of collagen, thereby improving the restoration of the area surrounding the excised tissue [11,15].

In addition to many studies reporting the significant bone healing efficacy of Tisseel, spontaenous bone healing should also be noted. Ihan Hren and Miljavec [14] assessed the spontaneous healing of bone defects in the mandible, and analyzed the association between the results and clinical parameters such as the size of the bone defect and the patient's age. According to the authors, small defects with a diameter of 20-30 $\mathrm{mm}$ were ultimately restored to about $97 \%$ of the normal bone density. Moreover, the study showed a statistically significant negative correlation between patient age and bone healing. In the cases of patients younger than 20 years, the final bone density was restored to $96 \%$ of normal bone, whereas in patients over 50 years, the recovery of bone defects only amounted to $80 \%$ of normal bone density after 1 year. De Oliveira et al. [15] designed an experiment to evaluate the effect of fibrin sealant in the recovery process of bone defects. They excluded female and young animals to minimize hormonal effects which could have a significant impact on bone regeneration.

One limitation of this study is that a 29-month-old female infant is likely to have very high spontaneous bone healing potential, meaning the efficacy of Tisseel in regeneration of bone defects might have been overrated. In addition, because it was difficult to round up patients with bone defects caused by dermoid cysts, we were only able to have follow-up observations on one patient. Further investigation into these products as surgical adjuvants that reduce postoperative complications, improve patient outcomes, and regenerate bone defects should be completed with many patients comprising different age groups.

To date, many cases of dermoid cysts accompanied by abnormalities in the underlying bone have been reported. However, in most studies, patients were followed up to evaluate the aesthetic outcomes of the surgical excision. Preoperative imaging was performed to identify bone abnormalities, but there was no postoperative CT to follow-up on correction of the bone defect. However, in this study, the dermoid cyst was fully excised, after which the fibrin sealant was applied. Complete recovery of the bone defect in the right zygoma was observed in a follow-up CT 6 months after the procedure.

\section{Conflict of interest}

No potential conflict of interest relevant to this article was reported. 


\section{ORCID iDs}

Hannara Park

https://orcid.org/0000-0003-4158-0489

Jaemin Seong

Hyochun Park

Hyeonjung Yeo

https://orcid.org/0000-0001-6019-8002

https://orcid.org/0000-0001-5705-7380

\section{References}

1. Tanwir A, Malik N, Javed G, et al. Dermoid cyst with no intracranial extension: a case report and literature review. Surg Neurol Int 2019;10:25.

2. Choi JS, Bae YC, Lee JW, et al. Dermoid cysts: epidemiology and diagnostic approach based on clinical experiences. Arch Plast Surg 2018;45:512-6.

3. Pushker N, Meel R, Kumar A, et al. Orbital and periorbital dermoid/epidermoid cyst: a series of 280 cases and a brief review. Can J Ophthalmol 2020;55:167-71.

4. Hoang VT, Trinh CT, Nguyen CH, et al. Overview of epidermoid cyst. Eur J Radiol Open 2019;6:291-301.

5. Sathananthan N, Moseley IF, Rose GE, et al. The frequency and clinical significance of bone involvement in outer canthus dermoid cysts. Br J Ophthalmol 1993;77:789-94.

6. Pollard ZF, Calhoun J. Deep orbital dermoid with draining sinus. Am J Ophthalmol 1975;79:310-3.

7. Hong S, Maruyama K, Hatanaka R, et al. Frontotemporal dermoid cyst with incomplete dermal sinus tract in an adult: a case report. Surg Neurol Int 2020;11:429.

8. Dave TV, Gupta Rathi S, Kaliki S, et al. Orbital and perior-

bital dermoid cysts: comparison of clinical features and management outcomes in children and adults. Eur J Ophthalmol 2020 Nov 16 [Epub]. https://doi.org/10.1177/ 1120672120964686.

9. Lee SS, Persing JA. Refinement in technique for pediatric dermoid cyst excision: technical note. Plast Reconstr Surg 2008;122:1059-61.

10. Wood J, Couture D, David LR. Midline dermoid cyst resulting in frontal bone erosion. J Craniofac Surg 2012;23: 131-4.

11. Kim WB, Kim SG, Lim SC, et al. Effect of Tisseel on bone healing with particulate dentin and plaster of Paris mixture. Oral Surg Oral Med Oral Pathol Oral Radiol Endod 2010;109:e34-40.

12. Kulber DA, Bacilious N, Peters ED, et al. The use of fibrin sealant in the prevention of seromas. Plast Reconstr Surg 1997;99:842-9.

13. Carmagnola D, Berglundh T, Lindhe J. The effect of a fibrin glue on the integration of Bio-Oss with bone tissue: a experimental study in labrador dogs. J Clin Periodontol 2002;29:377-83.

14. Ihan Hren N, Miljavec M. Spontaneous bone healing of the large bone defects in the mandible. Int J Oral Maxillofac Surg 2008;37:1111-6.

15. de Oliveira CTB, Leonel BC, de Oliveira AC, et al. Effects of fibrin sealant and bone fragments on defect regeneration performed on rat tibiae: an experimental study. J Mech Behav Biomed Mater 2020;104:103662. 